\title{
Two New Inherited Defects of the Thyroxine-binding Globulin (TBG) Molecule Presenting as Partial TBG Deficiency
}

\author{
Junta Takamatsu, ${ }^{\star}$ Samuel Refetoff, ${ }^{*}$ Myriam Charbonneau, ${ }^{\star}$ and Jean H. Dussault* \\ *Thyroid Study Unit, Departments of Medicine and Pediatrics, The University of Chicago, School of Medicine, Chicago, Illinois 60637; \\ and ${ }^{\ddagger}$ Laval University School of Medicine, and The Quebec Network of Genetic Medicine, Quebec City, Quebec; G1V-4G2 Canada
}

\begin{abstract}
Serum-denatured TBG (dnTBG) measured in 32 families deficient in native TBG (nTBG) was undetectable in all subjects with complete nTBG deficiency and was high in 2 of 16 families with partial nTBG deficiency. nTBG (in mean micrograms per decaliter \pm SD) in members of the Quebec and Montreal families, respectively, were: $258 \pm 54$ and 230 in affected men, $747 \pm 190$ and $927 \pm 90$ in affected women, and $1568 \pm 151$ and $1300 \pm 195$ in unaffected relatives. Corresponding mean dnTBG levels were: $14.3 \pm 2.9$ and 21.3 in affected men, 8.6 \pm 1.0 and 11.6 \pm 3.1 in affected women, and $<2.1$ and $<2.6$ in unaffected relatives. All were euthyroid with normal free thyroxine and thyrotropin levels. In comparison to common type TBG, TBG-Quebec was more heat labile by $10^{\circ} \mathrm{C}$ and TBG-Montreal by $12^{\circ} \mathrm{C}$. The degree of dnTBG elevation and nTBG lability at $37^{\circ} \mathrm{C}$ were correlated ( $r$ $=0.99$ ). . Isoelectric focusing showed cathodal shift of all TBG bands: TBG-Quebec by 0.06 isoelectric points (pI) and TBGMontreal by 0.02 pI. These two TBG variants represent different mutations most likely affecting the polypeptide chain of the molecule. Their inheritance is $\mathrm{X}$-chromosome linked. The instability of these TBGs at $37^{\circ} \mathrm{C}$ may lead to more rapid degradation in vivo resulting in low nTBG and high dnTBG concentrations in serum.
\end{abstract}

\section{Introduction}

Thyroxine-binding globulin (TBG) ${ }^{1}$, an acidic glycoprotein of hepatic origin, is the major thyroid hormone transport protein, carrying $\sim 75 \%$ of circulating thyroxine (T4) (1-3). Thus, significant changes in serum TBG concentration are associated with proportional alterations in serum T4 level, which can be erroneously interpreted as indicative of thyroid gland dysfunction.

Presented in part at the 68th Annual Meeting of the Endocrine Society, June 27th, 1986 in Anaheim, CA. Address correspondence to Dr. Refetoff, Thyroid Study Unit, Box 138, The University of Chicago, 5841 South Maryland Avenue, Chicago, IL 60637.

Received for publication 12 May 1986 and in revised form 15 September 1986.

1. Abbreviations used in this paper: CAP, capacity; dnTBG, denatured thyroxine-binding globulin; $\mathrm{FT}_{4} \mathrm{I}$, free T4 index; IEF, isoelectric focusing; nTBG, native TBG; RP-TBG, reduced and pyridylethylated TBG; T3, 3,3',5-triiodothyronine; T4, thyroxine; TBG-A, aboriginal type TBG; TBG-C, common type TBG; TBG-Quebec and TBG-Montreal, inherited variants described in detail in this paper; TBG-Gary, a heat labile variant TBG with low affinity for $\mathrm{T}_{4}$; TBG-S, isoelectric focusing variant TBG with cathodal or "slow" shift; $\operatorname{Tr}$ T3, total reverse T3; TSH, thyrotropin; TT3, total T3.

J. Clin. Invest.

(C) The American Society for Clinical Investigation, Inc.

0021-9738/87/03/0833/08 $\$ 1.00$

Volume 79, March 1987, 833-840
From the first recognition of a familial TBG abnormality in 1959 (4) and until 1980, studies of TBG in subjects with inherited TBG deficiency and excess failed to show physical or chemical changes of the molecule or alterations in the disappearance rate of labeled purified TBG given to the subjects (5-7). On this basis, it has been postulated that these $\mathrm{X}$-chromosome-linked TBG abnormalities represent either mutations of a gene regulating TBG synthesis (6) or subtle structural gene mutations that indirectly affect the rate of TBG synthesis (1). In 1981, Dick and Watson (8) reported that $\sim 40 \%$ of Australian Aborigines have low serum T4 concentration due to a variant TBG (TBGA) with low affinity for T4. The same year, Daiger et al. (9) reported another TBG with cathodal shift on isoelectric focusing (TBG-S) in 5-10\% of subjects of African or Oceanian origin. The demonstration of X-chromosome-linked inheritance of these variant TBGs with a preponderance in specific ethnic groups (9-11) and further studies of their properties (11-13) suggested that the structural gene of the molecule is located on the X-chromosome.

We recently described in a Caucasian family the occurrence of yet another variant TBG (TBG-Gary), which was extremely heat labile and weakly bound T4 (14). The finding that carriers of TBG-Gary had elevated levels of denatured TBG (dnTBG) in serum, not usually encountered in association with thyroidal or nonthyroidal illnesses (15), led us to measure dnTBG in sera from 32 unrelated families with inherited TBG deficiency. High dnTBG levels were thus found in serum samples from 2 of 16 families with partial TBG deficiency. Further studies revealed that these two families had variant TBGs with alterations in both stability and isoelectric focusing (IEF) patterns. These findings suggest that the inherited, nonethnic form of TBG deficiency comprises a number of genetic defects, some caused by structural gene mutations.

\section{Methods}

Serum samples. Sera were collected from subjects belonging to 32 unrelated families with inherited TBG deficiency. In 16 families, the TBG deficiency was complete and in the remaining 16 , it was partial. Complete deficiency was defined as the absence of detectable TBG in $10 \mu \mathrm{l}$ of serum measured by a radioimmunoassay (RIA) specific for native TBG (nTBG) (15) with a sensitivity of $0.1-0.2 \mathrm{ng}$ TBG (displacement of $>10 \%$ of antibody bound ${ }^{125}$ I-TBG in the absence of competitor). Thus, by this definition any serum with $\mathrm{nTBG}<2 \mu \mathrm{g} / \mathrm{dl}$ or $<0.125 \%$ the mean normal concentration of $1600 \mu \mathrm{g} / \mathrm{dl}$ was considered to be completely TBG deficient. Four of these families have been previously reported $(5,16,17)$ and eight with complete and four with partial TBG deficiency, were cases identified through the province of Quebec neonatal screening program in Canada.

Serum samples from an Australian Aborigine man with TBG-A (13) a Black-American man with TBG-S (11), and a man with TBG-Gary (14) were used for comparison. Use was also made of a serum pool (P24) containing more than 100 samples with values of nTBG, total T4 (TT4), free T4 index ( $\left.\mathrm{FT}_{4} \mathrm{I}\right)$, total 3,3',5-triiodothyronine (TT3), and thyrotropin 
(TSH) within a standard deviation of the normal mean and an IEF pattern of the common type TBG (TBG-C).

Chemicals. ${ }^{125} \mathrm{I}-\mathrm{T} 4$ with specific activity of $1250 \mu \mathrm{Ci} / \mu \mathrm{g}$ was obtained from New England Nuclear, (Boston, MA). L-T4 and $\gamma$-globulin-free bovine serum albumin (BSA) were purchased from Sigma Chemical Co. (St. Louis, MO). Acrylamide (ultra pure) was from Bethesda Research Laboratories (Gaithersburg, MD); and $N, N^{\prime}$ methylenebisacrylamide, ammonium persulfate, $N, N, N^{\prime}, N^{\prime}$-tetramethyl ethylenediamine from BioRad Laboratories (Richmond, CA). Ampholines were obtained from Pharmacia Fine Chemicals (Piscataway, NJ) and electrode fluids from Serva Fine Biochemicals, Inc. (Garden City Park, NY). Barbital buffer, type B-2 (pH 8.6) was from EM Diagnostic Systems, Inc. (Gibbstown, NJ) and resin (Rexyn 201) from Fisher Scientific Co. (Fair Lawn, NJ). Goat anti-rabbit $\gamma$-globulin was purchased from Antibodies Inc., (Davis, CA). The preparation of purified TBG, dnTBG by reduction and pyridylethylation (RP-TBG), and antisera for nTBG and RP-TBG were described in detail elsewhere (15).

RIAs and tests of thyroid function. Concentrations of TT4, TT3, total reverse $\mathrm{T} 3\left(\mathrm{TrT}_{3}\right)$, and TSH in serum were measured by RIAs used routinely in our clinical diagnostic laboratory. Levels of nTBG and dnTBG in serum were determined by specific RIAs as previously described (15). In addition, the T4-binding capacity of nTBG (nTBG-CAP) was measured at saturation by the single $\mathrm{T} 4$ load-ion-exchange resin method (18) and the $\mathrm{FT}_{4} \mathrm{I}$ was calculated from the TT4 concentration and the resin T4 uptake ratio (19). The titers of thyroglobulin and thyroid microsomal antibodies were measured by the hemagglutination technique (20). Table I gives normal ranges.

Parameters of T4 binding to TBG. The method used was essentially that previously described (13). Serum samples were diluted with $75 \mathrm{mM}$ barbital buffer ( $\mathrm{pH} 8.6$ ) to a concentration of $\sim 1 \mu \mathrm{g} \mathrm{nTBG} / \mathrm{ml}(16 \mathrm{nM})$, before addition of ${ }^{125} \mathrm{I}-\mathrm{T} 4$ and $0.039-1.25 \mathrm{ng}$ unlabeled L-T4/ml. Nonspecific binding was determined in the presence of $500 \mathrm{ng}(640 \mathrm{nM})$ unlabeled T4. After equilibration at $0^{\circ} \mathrm{C}$, TBG-bound T4 was separated from free or albumin-bound $\mathrm{T} 4$ by ion-exchange resin beads and the association constants $\left(K_{\mathrm{a}}\right)$ and maximal binding capacity were calculated by the method of Scatchard (21). The lack of influence by other proteins in serum at these dilutions has been confirmed (13).

Heat and acid denaturation of $n T B G$. The rate of heat denaturation of nTBG was determined after measurement of the residual nTBG by RIA in serum samples incubated for various periods of time at different temperatures using a water bath with a precision of $\pm 0.1^{\circ} \mathrm{C}$. Serum samples were first diluted in $10 \mathrm{mM}$ sodium phosphate (pH 7.4 buffered), and $150 \mathrm{mM}$ saline phosphate-buffered saline containing $1 \% \mathrm{BSA}$. When incubated for $>2 \mathrm{~h}$ at relatively low temperatures, the samples were first sterilized by passage through a $0.45-\mu \mathrm{m}$ Millipore filter. The $t_{1 / 2}$ of $\mathrm{nTBG}$ was calculated by the least-square analysis of the logarithm of residual nTBG concentrations and the corresponding times of incubation (10). The effect of added T4 on the heat denaturation of nTBG was studied as previously described (15).

Residual nTBG was measured by RIA after timed exposure of serum samples to various pHs to determine its stability to acid. $10 \mu \mathrm{l}$ of serum was added to a $\mathrm{ml}$ of $25 \mathrm{mM}$ sodium acetate buffers at $\mathrm{pH} 5.0,4.5,4.0$, 3.75, and 3.5, followed by incubation for $\mathbf{4 0} \mathrm{min}$ at room temperature. The solutions were neutralized by the addition of $200 \mu \mathrm{l} 2 \mathrm{M}$ Tris buffer (pH 7.8). Measurement of the $\mathrm{pH}$ in some samples indicated that the serum exerted buffering effect at the lowest $\mathrm{pH}$ so that the final $\mathrm{pH}$ could have been up to $0.2 \mathrm{pH}$ higher, and after neutralization, up to $0.2 \mathrm{pH}$ lower. However, further dilution with PBS-1\% BSA before RIA brought the $\mathrm{pH}$ to 7.4.

IEF. Horizontal, 1-mm-thick, slab gels of $5 \%$ total acrylamide $(0.125 \%$ bis-acrylamide) containing $8 \%$ glycerol and $6.7 \%$ ampholines of pH 4.24.9 were used for IEF. They were polymerized in a capillary casting mold with $250 \mu \mathrm{l}$ of $0.1 \mathrm{M}$ ammonium persulfate and $34 \mu \mathrm{l} N, N, N^{\prime}, N^{\prime}$-tetramethyl ethylenediamine $/ 30 \mathrm{ml}$ of gel solution. After applying anode fluid 3 and cathode fluid 10 , gels were prefocused for 30-60 min at a constant power of $12 \mathrm{~W}$. Serum samples of $8 \mu \mathrm{l}$ each were applied after their incubation with $10 \mathrm{nCi}$ of ${ }^{125} \mathrm{I}-\mathrm{T} 4$, for at least $1 \mathrm{~h}$ at room temperature. Electrodes were placed $9 \mathrm{~cm}$ apart and the samples were focused at con- stant power, starting with 8-12 W for a total of $3,500 \mathrm{~V} / \mathrm{h}$. At the end of the run, gels were immediately dried for $1 \mathrm{~h}$ at $60^{\circ} \mathrm{C}$, covered with plastic wrap, and exposed to Kodak X-AR5 film with an intensifying screen for $12-24 \mathrm{~h}$ at $-70^{\circ} \mathrm{C}$.

All grouped data are expressed as the mean $\pm \mathrm{SD}$, and were analyzed by the $t$ test (22). Regression lines were computed by the method of least squares (22). Statistical significance is defined as $P<0.05$.

\section{Results}

dnTBG in subjects with inherited TBG deficiency. dnTBG was measured in serum samples from at least two individuals from each of the 32 families, one hemizygous TBG-deficient male and one heterozygous female carrier. In many instances, sera from other affected family members and an unaffected relative were also examined so that a total of 91 subjects had dnTBG determinations. dnTBG was not detectable $(<2 \mu \mathrm{g} / \mathrm{dl})$ in any of the serum samples from subjects with complete nTBG deficiency $(<2 \mu \mathrm{g} / \mathrm{dl})$. It was within the normal range $(<2-8 \mu \mathrm{g} /$ dl) in heterozygous affected and unaffected members from the 16 families with complete deficiency and in all members of 14 of the 16 families with partial nTBG deficiency tested. Because clearly elevated dnTBG values were found in two families with partial nTBG deficiency, further studies were undertaken to analyze all available serum samples from these families and to characterize their TBG. One family was from the vicinity of Quebec (TBG-Quebec) and the other was living in Montreal (TBG-Montreal). Both families were of French Canadian extraction. As their pedigrees in Fig. 1 show, based on the degree of dnTBG elevation and nTBG depression, the inheritance appears to be X-chromosome linked.

Thyroid function tests. Tables I and II summarize the data for the Quebec and the Montreal family, respectively. The concentrations of nTBG (RIA or CAP), and TT4 were lowest and dnTBG highest in hemizygous affected subjects and intermediate in heterozygous carriers as compared to unaffected relatives, without overlap between individual values among these three groups. Also, mean values of TT3 and TrT3 for hemizygous affected subjects in the Quebec family were significantly lower than those of unaffected relatives. In heterozygous carriers, the mean value of TT3 and TrT3 in the Quebec family and that of TrT3 but not TT3 in the Montreal family were significantly lower than those of nonaffected relatives. Values of TBG-CAP were congruent to those measured by RIA in subjects from all groups. None of the subjects had a goiter and they were clinically euthyroid with levels of $\mathrm{FT}_{4} \mathrm{I}$ and TSH within the normal range and no significant differences of their mean values among the three groups. None had detectable circulating antibodies to thyroglobulin or thyroid microsomes.

Immunologic properties of serum TBGs. Serum samples from affected hemizygous subjects of both families were serially diluted and tested for their ability to inhibit binding of ${ }^{125}$ I-nTBG (Fig. 2) and ${ }^{125}$ I-RP-TBG (not shown) to their respective polyclonal antibodies as compared with a normal serum pool, and a standard of purified TBG preparation. No immunologic differences could be detected by this technique. These results are in agreement with the lack of discrepancies in nTBG estimated by RIA and/or CAP.

Binding affinity of the $n T B G$ for T4. The $K_{\mathrm{a}}$ of nTBG for T4 was determined in serum from each member of both families and Tables I and II give the results. nTBGs from hemizygous affected men showed only a slight reduction in $K_{\mathrm{a}}$. Nevertheless, the mean value was significantly lower than in unaffected rela- 
A

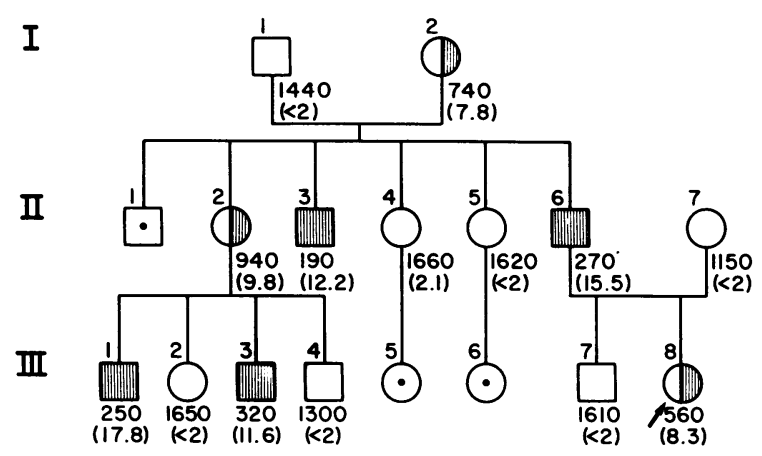

B

I

II

III

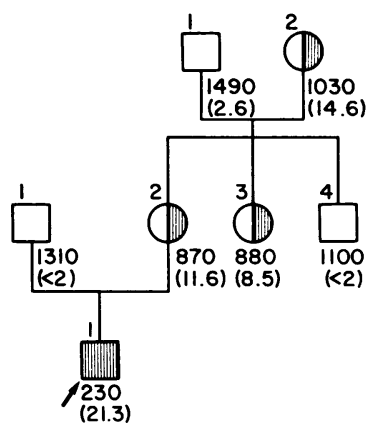

Figure 1. Pedigrees of the Quebec $(A)$ and Montreal $(B)$ families. Subjects are identified by generation (in Roman numeral) and a number to the left and above each symbol. Numbers under each symbol are the concentrations of nTBG and dnTBG (in brackets) in micrograms per decaliter of serum. Open boxes, unaffected male; hatched boxes, hemizygous affected male; open circles, unaffected female; partially hatched circles, heterozygous affected females; dot, unaffected male or female not studied in detail; arrow, propositus.

tives. This finding was consistent in all subjects and reproducible in separate determinations with $K_{\mathrm{a}}$ values of $0.82,0.73$, and 0.71 $\times 10^{10} \mathrm{M}^{-1}$ in one serum (Table I) and $0.88,0.91$, and 0.61 $\times 10^{10} \mathrm{M}^{-1}$ measured in another serum (Table II), each at three different dilutions $(1: 200,1: 400$, and 1:800, respectively). Sera from both affected and unaffected individuals in which nTBG was denatured by heat treatment showed no demonstrable T4binding activity.
Denaturation of nTBG by heat and by acid. When sera from members of the Quebec family were incubated at $56^{\circ} \mathrm{C}$, the four samples from the hemizygous affected men lost $88-92 \%$ of their basal nTBG within the first $4 \mathrm{~min}$ (Fig. 3). Sera from the three heterozygous affected subjects lost $15-59 \%$ of their basal nTBG within the same time period when exposed to $56^{\circ} \mathrm{C}$. This corresponds to $120-240 \mu \mathrm{g} \mathrm{nTBG/dl} \mathrm{denatured} \mathrm{or} \mathrm{slightly} \mathrm{more}$ than half the amount of nTBG denatured from serum of the hemizygous men (180-295 $\mu \mathrm{g} / \mathrm{dl})$. The residual 340-800 $\mu \mathrm{g}$ nTBG/dl was degraded with a $t_{1 / 2}$ ranging from 45 to $50 \mathrm{~min}$, which is similar to that of unaffected family members (37-53 min) (Fig. 3). Similar data were obtained in sera from members of the Montreal family, although the nTBG component with rapid heat denaturation in heterozygous carriers was smaller, representing $10-25 \%$ of their basal nTBG (Fig. 3). The $t_{1 / 2}$ of the residual nTBG ranged from 33 to $47 \mathrm{~min}$ in these sera, which was similar to that of 36-52 min in the three unaffected relatives. As shown below, the heat labile component of nTBG in heterozygous carriers could be identified by IEF as representing the variant $\mathrm{nTBG}$.

Because of the very rapid denaturation at $56^{\circ} \mathrm{C}$ of nTBG of affected subjects from both families, the rates of nTBG denaturation were determined at different temperatures. As Fig. 4 shows, nTBG in a hemizygous affected subject (III-1) from TBGQuebec was less heat labile by $\sim 2^{\circ} \mathrm{C}$ as compared with TBGMontreal. However, in comparison to TBG-C, TBG-Quebec, and TBG-Montreal were more labile by $10^{\circ}$ and $12^{\circ} \mathrm{C}$, respectively. Their temperature lability was further compared with other variant TBGs, showing that TBG-Quebec was less labile by $\sim 5^{\circ} \mathrm{C}$ than the variant TBG-Gary (14) and more labile than TBG-A by $8^{\circ} \mathrm{C}$ and TBG-S by $9^{\circ} \mathrm{C}$ (Fig. 4).

When sera were incubated at $37^{\circ} \mathrm{C}$, no denaturation could be demonstrated in TBG-C or TBG-A over a period of $6 \mathrm{~d}$, while TBG-Quebec (II-6) and TBG-Montreal (III-1) were denatured with a $t_{1 / 2}$ of 3.6 and $2.9 \mathrm{~d}$, respectively (Fig. 5). Addition of excess T4 (18 $\mu \mathrm{g} / \mu \mathrm{g}$ TBG) to serum samples from hemizygous affected subjects from both families partially protected the heat denaturation of their nTBG at $56^{\circ} \mathrm{C}$ (Fig. 6).

Fig. 7 shows the effect of acid treatment on the denaturation of nTBG. As in the case of heat, TBG-Montreal (III-1) was more susceptible to denaturation than TBG-Quebec (hemizygous affected). However, at pHs lower than 4.5, the latter appeared to be relatively more stable than TBG-C. This behavior of TBGQuebec was observed in all four hemizygous affected subjects of the family.

The defective nTBG from both families lost by in vitro heat

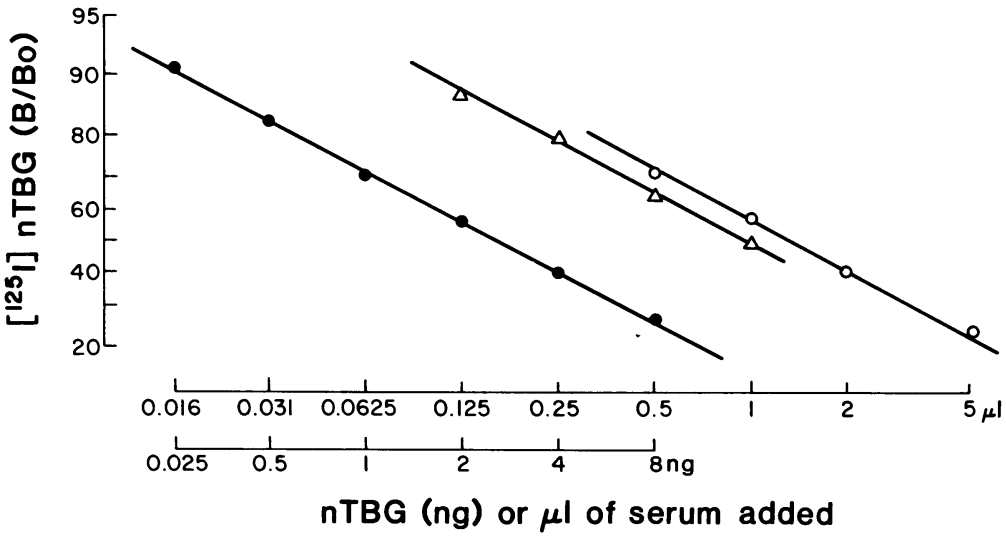

Figure 2. Immunoreactivity of TBG-Quebec (open triangles, II-6) and TBG-Montreal (open circles, III-1) in the nTBG RIA. Sera from hemizygous affected subjects were serially diluted before assay. Data are plotted as the logit$\log$ of the percentage of the ratio of $\left[{ }^{125} \mathrm{I}\right] \mathrm{nTBG}$ bound to the antiserum in the presence $(B)$ and absence $(B o)$ of sample. Note the parallelism with serial dilutions of a normal serum pool (P24) (filled circles) containing TBG-C. 
Table I. Thyroid Function Tests in Members of the Quebec Family

\begin{tabular}{|c|c|c|c|c|c|c|c|c|c|c|}
\hline \multirow[b]{2}{*}{ Subject } & \multirow[b]{2}{*}{ Sex } & \multicolumn{3}{|l|}{ nTBG } & \multirow{2}{*}{$\begin{array}{l}\text { dnTBG } \\
\text { RIA }\end{array}$} & \multirow[b]{2}{*}{ TT4 } & \multirow[b]{2}{*}{ TT3 } & \multirow[b]{2}{*}{ TrT3 } & \multirow[b]{2}{*}{$\mathrm{FT}_{4} \mathbf{I}$} & \multirow[b]{2}{*}{ TSH } \\
\hline & & $K_{\mathrm{a}}$ for $\mathrm{T} 4$ & CAP & RIA & & & & & & \\
\hline & & $\times 10^{-10} \mathrm{M}^{-1}$ & $\mu g T 4 / d l$ & $\mu g / d l$ & $\mu g / d l$ & $\mu g / d l$ & $n g / d l$ & $n g / d l$ & & $\mu U / m l$ \\
\hline \multicolumn{11}{|c|}{ Hemizygous affected } \\
\hline II-3 & $\mathbf{M}$ & 0.90 & 2.8 & 190 & 12.2 & 3.1 & 65 & 14.4 & 8.0 & 2.2 \\
\hline II-6 & $\mathbf{M}$ & $0.77^{*}$ & 4.7 & 270 & 15.5 & 3.9 & 82 & 19.1 & 9.0 & 2.1 \\
\hline III-1 & $\mathbf{M}$ & 0.73 & 4.1 & 250 & 17.8 & 2.8 & 78 & 12.9 & 7.0 & 3.3 \\
\hline III-3 & $\mathbf{M}$ & 0.69 & 5.1 & 320 & 11.6 & 3.8 & 96 & 14.5 & 7.6 & 2.5 \\
\hline Mean & & $0.77^{\$ 8}$ & $4.18^{\ddagger 8}$ & $258^{\ddagger 8}$ & $14.3^{\neq 8}$ & $3.40^{\neq 8}$ & $80.5^{\ddagger}$ & $15.2^{\ddagger}$ & 7.90 & 2.53 \\
\hline \pm SD & & 0.09 & 1.00 & 54 & 2.9 & 0.54 & 12.7 & 2.7 & 0.84 & 0.54 \\
\hline \multicolumn{11}{|c|}{ Heterozygous carriers } \\
\hline $\mathrm{I}-2$ & $F$ & 1.21 & 11.9 & 740 & 7.8 & 4.9 & 95 & 14.5 & 6.7 & 1.4 \\
\hline II-2 & $\mathbf{F}$ & 1.00 & 12.3 & 940 & 9.8 & 6.3 & 88 & 23.8 & 8.4 & 0.7 \\
\hline III-8 & $F$ & 0.95 & 7.3 & 560 & 8.3 & 4.6 & 81 & 20.3 & 8.4 & 3.5 \\
\hline Mean & & 1.05 & $10.50^{\ddagger}$ & $747^{\ddagger}$ & $8.6^{\ddagger}$ & $5.27^{\ddagger}$ & $88.0^{\ddagger}$ & $19.5^{\ddagger}$ & 7.83 & 1.87 \\
\hline \pm SD & & 0.14 & 7.72 & 190 & 1.0 & 0.91 & 7.0 & 4.7 & 0.98 & 1.46 \\
\hline \multicolumn{11}{|c|}{ Unaffected blood relatives } \\
\hline II-4 & $F$ & 1.29 & 23.5 & 1660 & $<2.1$ & 9.9 & 141 & 27.7 & 8.6 & 1.5 \\
\hline II-5 & $\mathbf{F}$ & 1.12 & 19.5 & 1620 & $<2$ & 6.9 & 94 & 26.0 & 6.8 & 1.0 \\
\hline III-2 & $\mathbf{F}$ & 0.96 & 22.5 & 1650 & $<2$ & 10.5 & 159 & 28.8 & 9.5 & 1.9 \\
\hline III-4 & $\mathbf{M}$ & 1.29 & 20.2 & 1300 & $<2$ & 9.6 & 164 & 24.2 & 9.4 & 1.8 \\
\hline III-7 & $\mathbf{M}$ & 0.92 & 20.5 & 1610 & $<2$ & 10.4 & 159 & 27.6 & 10.2 & 5.0 \\
\hline Mean & & 1.12 & 21.24 & 1568 & $<2.1$ & 9.46 & 143.4 & 26.9 & 8.90 & 2.24 \\
\hline$\pm \mathrm{SD}$ & & 0.18 & 1.68 & 151 & & 1.48 & 29.0 & 1.8 & 1.30 & 1.58 \\
\hline \multicolumn{11}{|c|}{ Related by marriage } \\
\hline $\mathrm{I}-1$ & $\mathbf{M}$ & 1.25 & 19.0 & 1440 & $<2$ & 9.8 & 112 & 33.4 & 9.7 & 3.7 \\
\hline II-7 & $\mathbf{F}$ & 1.09 & 16.5 & 1150 & $<2$ & 7.3 & 126 & 20.7 & 8.1 & 2.0 \\
\hline Normal & & $0.70-1.35$ & $14-25$ & $1100-2100$ & $<2-8$ & $5.0-12.0$ & $90-170$ & $14.5-30$ & $6.5-10.5$ & $0.4-5.0$ \\
\hline
\end{tabular}

* Mean value of three separate determinations ranging from 0.71 to 0.82 . ${ }^{\ddagger} P<0.05-0.0001$ as compared with the respective mean values of normal blood relatives. ${ }^{8} P<0.05-0.005$ as compared with the respective mean value of heterozygous carriers.

Table II. Thyroid Function Tests in Members of the Montreal Family

\begin{tabular}{|c|c|c|c|c|c|c|c|c|c|c|}
\hline \multirow[b]{2}{*}{ Subject } & \multirow[b]{2}{*}{ Sex } & \multicolumn{3}{|l|}{ nTBG } & \multirow[b]{2}{*}{$\begin{array}{l}\text { dnTBG } \\
\text { RIA }\end{array}$} & \multirow[b]{2}{*}{ TT4 } & \multirow[b]{2}{*}{ TT3 } & \multirow[b]{2}{*}{ TrT3 } & \multirow[b]{2}{*}{$\mathrm{FT}_{4} \mathrm{I}$} & \multirow[b]{2}{*}{ TSH } \\
\hline & & $K_{\mathrm{A}}$ for T4 & CAP & RIA & & & & & & \\
\hline & & $\times 10^{-10} M^{-1}$ & $\mu g T 4 / d l$ & $\mu g / d l$ & $\mu g / d l$ & $\mu g / d l$ & $n g / d l$ & $n g / d l$ & & $\mu U / m l$ \\
\hline \multicolumn{11}{|c|}{ Hemizygous affected } \\
\hline III-1 & $\mathbf{M}$ & $0.80^{*}$ & 3.6 & 230 & 21.3 & 3.1 & 89 & 13.2 & 8.3 & 2.7 \\
\hline \multicolumn{11}{|c|}{ Heterozygous carriers } \\
\hline $\mathrm{I}-2$ & $F$ & 0.98 & 13.0 & 1030 & 14.6 & 5.9 & 92 & 23.1 & 7.6 & 2.2 \\
\hline II-2 & $\mathbf{F}$ & 1.11 & 12.3 & 870 & 11.6 & 5.7 & 88 & 19.4 & 7.6 & 1.5 \\
\hline III-3 & $\mathbf{F}$ & 0.99 & 13.5 & 880 & 8.5 & 6.2 & 117 & 21.3 & 7.9 & 2.7 \\
\hline Mean & & 1.03 & $12.93^{\ddagger}$ & $927^{\ddagger}$ & $11.6^{\ddagger}$ & $5.93^{\ddagger}$ & 99.0 & $21.3^{\ddagger}$ & 7.70 & 2.13 \\
\hline \pm SD & & 0.07 & 0.60 & 90 & 3.1 & 0.25 & 15.7 & 1.9 & 0.17 & 0.60 \\
\hline \multicolumn{11}{|c|}{ Unaffected relatives } \\
\hline $\mathrm{I}-1$ & $\mathbf{M}$ & 1.24 & 18.8 & 1490 & 2.6 & 7.2 & 133 & 25.0 & 7.3 & 1.9 \\
\hline II-1 & $\mathbf{M}$ & 0.95 & 18.3 & 1310 & $<2$ & 8.4 & 144 & 24.7 & 8.8 & 2.9 \\
\hline II-4 & $\mathbf{M}$ & 0.96 & 17.8 & 1100 & $<2$ & 8.9 & 118 & 26.2 & 9.5 & 4.9 \\
\hline Mean & & 1.05 & 18.30 & 1300 & $<2.6$ & 8.17 & 131.7 & 25.3 & 8.53 & 3.23 \\
\hline$\pm \mathrm{SD}$ & & 0.16 & 0.50 & 195 & & 0.87 & 13.1 & 0.79 & 1.12 & 1.53 \\
\hline
\end{tabular}

Table I gives normal ranges. ${ }^{*}$ Mean value of three separate determinations ranging from 0.61 to $0.91{ }^{\ddagger} P<0.05-0.002$ as compared with the respective mean values of unaffected relatives. 

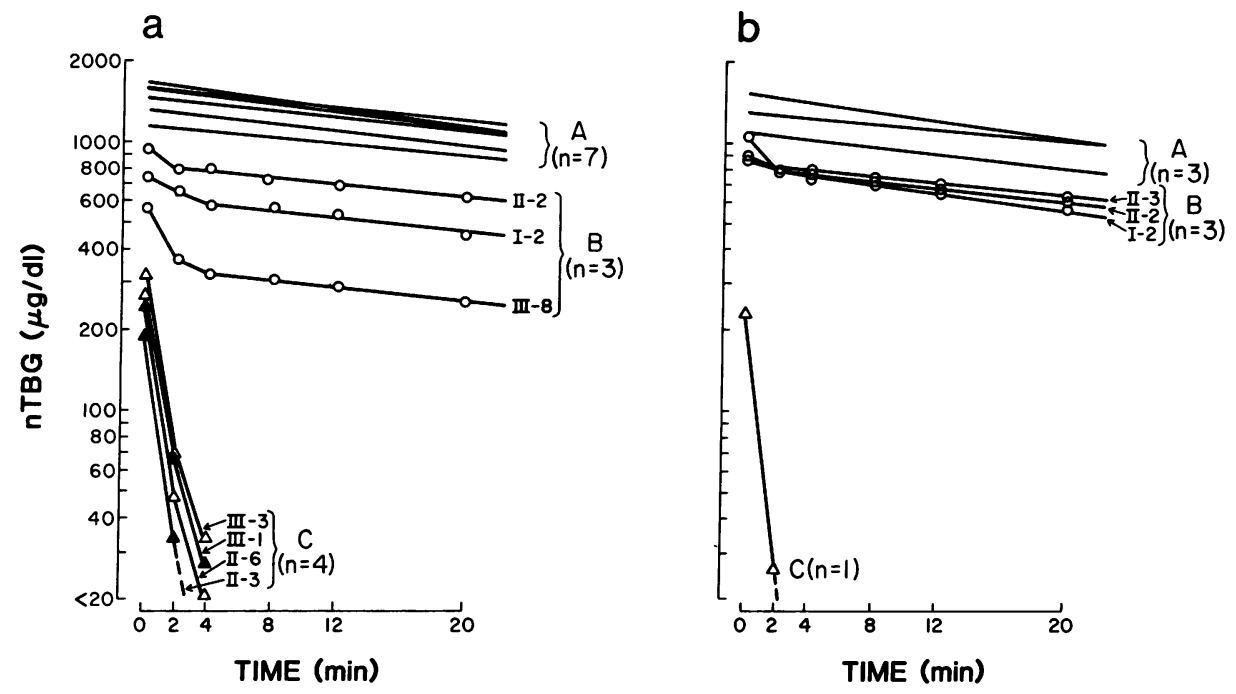

Figure 3. Rates of nTBG denaturation at $56^{\circ} \mathrm{C}$. Appropriately diluted serum samples from all members of Quebec $(a)$ and Montreal $(b)$ families were exposed to $56^{\circ} \mathrm{C}$ for various times followed by measurement of $n T B G$ by RIA. Note the rapid denaturation of $n T B G$ in affected men $(C)$ as compared with the unaffected relatives $(A)$, and the two components of the heat inactivation curves in affected, heterozygous women $(B)$. Individual subjects are identified as in Fig. 1.

or acid treatment was quantitatively recovered as dnTBG when measured by its specific RIA, in agreement with data on TBG$C$ and all other variant TBGs so far studied (11, 13-15).

IEF patterns. The multibanded pattern of TBG-C comprises three major bands with isoelectric points (pls) of $4.35,4.42$, and 4.50. The same pattern was obtained in unaffected members of the two families. As Fig. 8 shows, hemizygous affected men from the Quebec family showed a 0.06-pl cathodal shift of all bands giving rise to an IEF pattern indistinguishable from that of TBGS. A lesser cathodal shift of $0.02 \mathrm{pl}$ was observed in the pattern of TBG-Montreal (hemizygous affected III-1). Heterozygous carrier women from both families had IEF patterns showing a mixture of their inherited variant TBG and TBG-C. This is clearly demonstrated in Fig. 8, when comparing IEFs of serum samples before and after exposure for $5 \mathrm{~min}$ to $56^{\circ} \mathrm{C}$, which allows the preferential denaturation of the variant TBG. It not only proves that heterozygous carriers have both the variant and common type TBG, but that the heat lability is an inherent property of the TBG molecule rather than the effect of extraneous serum factors.

\section{Discussion}

As is the case with other known forms of inherited TBG abnormalities or variants $(1,2,7,9-13)$, the TBG defects herein described appear to be transmitted in an X-chromosome-linked fashion. Indeed, in both families, the lowest nTBG levels and highest dnTBG levels were found in the five affected men while values in the six affected women were intermediate to those of affected men and unaffected relatives with no overlap of individual values between these three groups. Evidence that all affected men were hemizygous, while affected women were heterozygous was obtained from data on heat denaturation and IEF of nTBG. Serum samples from all affected women contained both the abnormal TBG and normal TBG-C. In contrast, affected men had exclusively either the abnormal TBG or TBG-C. In agreement with random $\mathrm{X}$-chromosome inactivation, sera from heterozygous affected women had approximately one-half the concentration of the variant TBG found in affected men and one-half the amount of TBG-C found in unaffected subjects. Data on the inheritance pattern is limited by the paucity of progeny from affected men. However, the one affected man (II-6) from the Quebec family had an unaffected son (III-7) and an affected heterozygous daughter (III-8), a finding compatible with of X-chromosome-linked inheritance.

Results of thyroid function tests and nTBG and dnTBG values were similar in both families. The abnormal nTBGs from both families had comparable reduction of affinity for T4. Also, the abnormal nTBGs and dnTBG of the two families were immunologically indistinguishable when competed respectively,
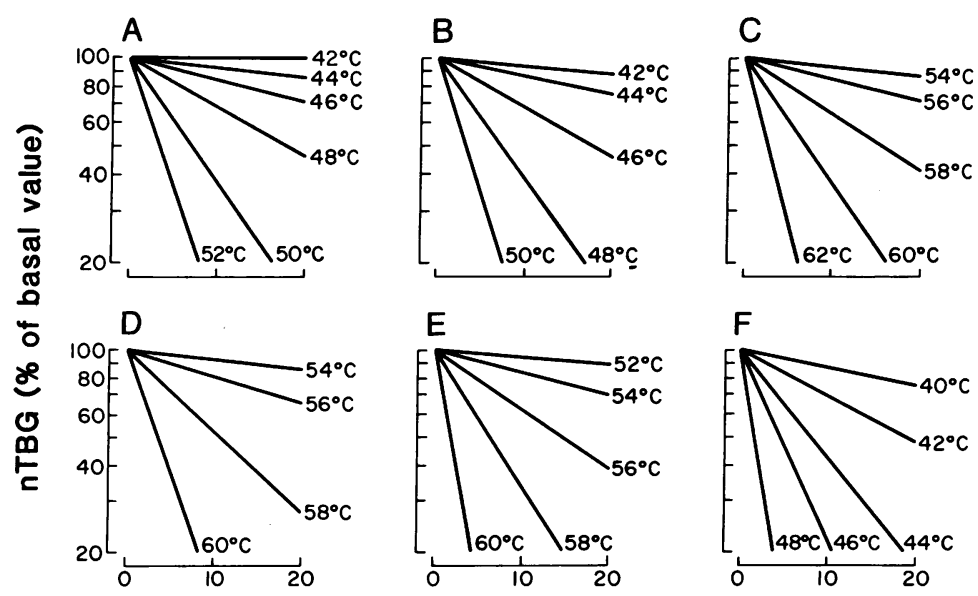

TIME (min)
Figure 4. Rates of nTBG denaturation at various temperatures of TBG-Quebec $(A)$ and TBG-Montreal $(B)$ as compared with TBG-C and three other variant types of TBG $(D$, TBG-S; $E$, TBG-A; and E, TBG-Gary). All studies were carried out on individual serum samples from hemizygous men Results are expressed as the percentage of nTBG concentration before heat treatment. 


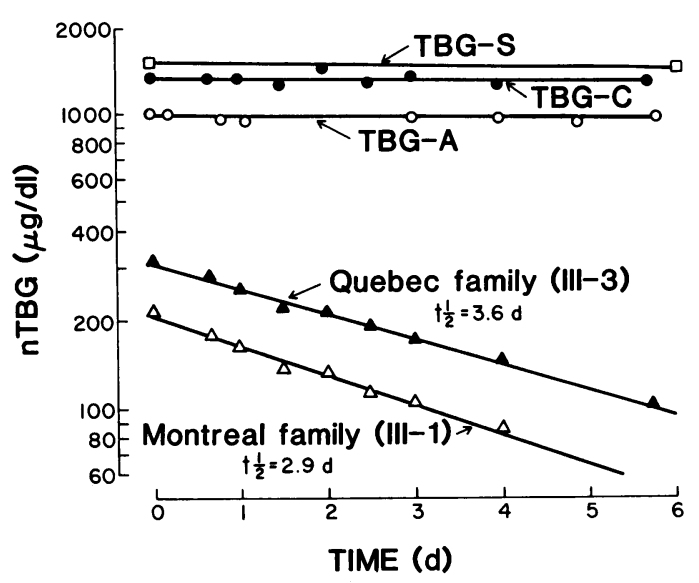

Figure 5. Rates of nTBG denaturation at $37^{\circ} \mathrm{C}$. Sterile serum samples were exposed to $37^{\circ} \mathrm{C}$ for various times followed by measurement of nTBG by RIA. Note the loss of nTBG in sera from hemizygous affected subjects from the Quebec and Montreal families, in contrast to the lack of denaturation in sera containing TBG-S, TBG-C, and TBGA. The $t_{1 / 2}$ of denaturation and the subject's identity are given next to each curve.

with the native and denatured forms of TBG-C for binding to polyclonal antibodies. However, denaturation studies showing greater heat lability of TBG-Montreal than TBG-Quebec at all temperatures suggested that the abnormal nTBGs were different in each family. TBG-Montreal was also more easily denatured than TBG-Quebec at pH 5 and 4.5 and T4 protected TBGMontreal to a lesser extent from heat denaturation. Another distinction between these two abnormal TBGs was their different pIs. The 0.06-pl cathodal shift of TBG-Quebec was more pronounced than that of TBG-Montreal of only $0.02 \mathrm{pl}$. In this respect, TBG-Quebec was indistinguishable from the TBG-S variant found with high frequency in American Blacks (9, $11,12)$.

To compare TBG-Quebec and TBG-Montreal with other known TBG variants in humans, data on nTBG concentration

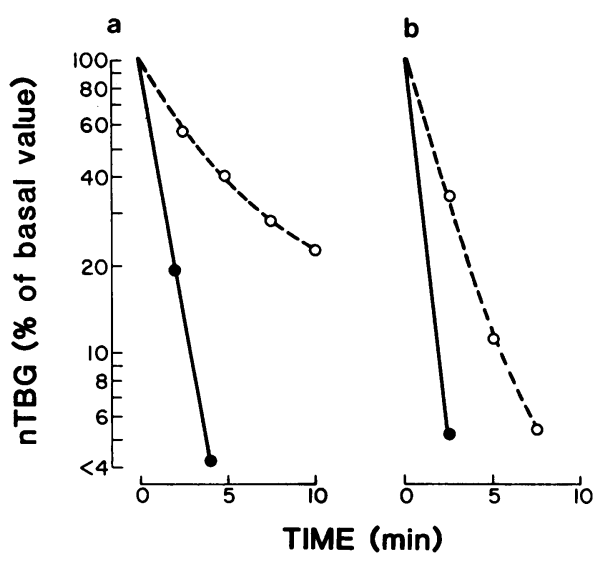

Figure 6. Effect of excess $\mathrm{T} 4$ on the denaturation of $\mathrm{nTBG}$ at $56^{\circ} \mathrm{C}$. Sera of hemizygous affected subjects from Quebec $(a)$ and Montreal (b) families were heated for various time periods in the absence and presence of added T4. Results are expressed as the percentage of the nTBG concentration before heat treatment. Note the protective effect of $T_{4}$ on the rates of heat denaturation of both nTBGs. Filled circles, $-\mathrm{T}_{4}$; open circles, $+\mathrm{T}_{4}(18 \mu \mathrm{g} / \mu \mathrm{g} \mathrm{TBG})$.

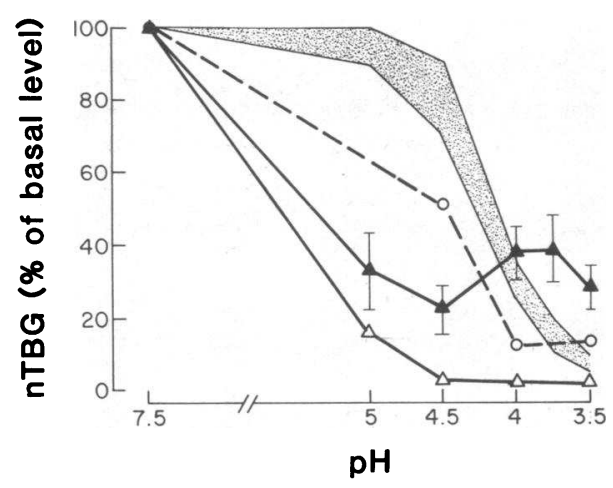

Figure 7. Acid denaturation of nTBG. Sera were incubated for $40 \mathrm{~min}$ at room temperature in buffer solutions of decreasing $\mathrm{pH}$. The solutions were neutralized and the residual nTBG was measured by RIA. Data are expressed as percentage of the nTBG concentration before exposure to acid pH. Results of four affected subjects from the Quebec family are plotted as mean \pm SD. TBG-C (stippled area, range); open circles, TBG-A; solid triangles, Quebec family, $n=4$ (hemizygous affected); open triangles, Montreal family (III-1).

and properties and dnTBG levels were summarized in Table III. An inverse correlation between serum nTBG concentrations and heat lability is apparent. Furthermore, only three types of TBG, which are instable at $37^{\circ} \mathrm{C}$ (TBG-Quebec, TBG-Montreal, and TBG-Gary) but not the other two variants, which are stable at $37^{\circ} \mathrm{C}$ (TBG-S and TBG-A), were associated with high levels of circulating dnTBG. The average concentration of the latter increased (260\% for TBG-Quebec, $390 \%$ for TBG-Montreal, and $1,000 \%$ for TBG-Gary) with the relative increase in heat lability of the respective nTBG at $37^{\circ} \mathrm{C}\left(t_{1 / 2}=3.6,2.9\right.$, and $0.13 \mathrm{~d}, r$ $=0.99$ ). Because dnTBG is generated from nTBG by heat treatment, we have previously suggested that the high endogenous dnTBG may reflect the more rapid in vivo degradation of the abnormal nTBG (14). This hypothesis, though not proven, is supported by the results from these two new families with abnormal TBGs. Reduced synthesis or secretion of the abnormal nTBGs and concomitant release by the liver of a precursor in the form of dnTBG cannot be ruled out. Indeed, poorly structured TBG could behave immunologically as dnTBG (23).

The variant TBGs had some overlapping properties (Table III). The cathodal IEF shifts of TBG-Quebec and TBG-S are identical but TBG-S is more stable, has normal affinity for T4 and carriers of TBG-S have normal dnTBG levels. TBG-A has an IEF pattern indistinguishable from TBG-C but has increased heat lability and reduced affinity for T4 and T3. Finally, TBGQuebec and TBG-Montreal have similar reduction in affinity for T4 and relatively small differences in heat lability but can be clearly differentiated by their IEF patterns. Thus, no single measurement that Table III lists could by itself differentiate among the six currently known types of TBG.

The precise alterations of the TBG molecule remain unknown. We favor the hypothesis of differences in the polypeptide chain, which in some instances result in secondary variation in the carbohydrate moieties, for the following reasons. In vitro deglycosylation of purified TBG-C has little, if any, effect on the heat lability of the molecule so that the observed heat lability of variant TBGs cannot be explained by even profound alterations in the carbohydrate chains. In contrast, the carbohydrate and 


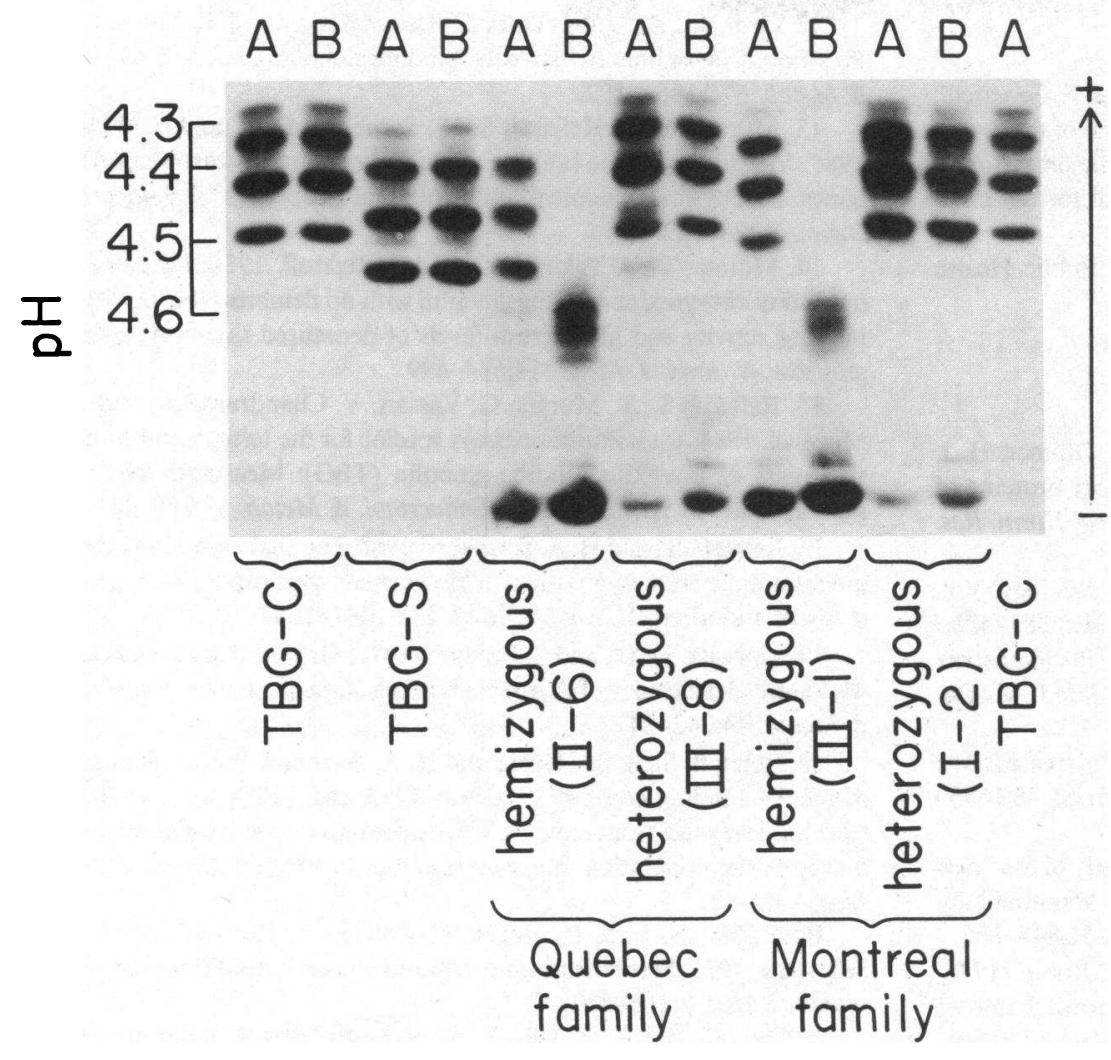

Figure 8. IEF of serum nTBG. Untreated $(A)$ or treated for $5 \mathrm{~min}$ at $56^{\circ} \mathrm{C}(B)$ sera were preincubated with ${ }^{125} \mathrm{I}-\mathrm{T} 4$, submitted to IEF, and the dried gel was radioautographed. Note preservation of ${ }^{125}$ I-T4-binding activity to the TBG-C and the IEF-variant (TBG-S) bands after exposure to heat. The variant TBGs, Quebec (II-6) and Montreal (III-1), have shifted bands that lost ${ }^{125} \mathrm{I}-\mathrm{T} 4-$ binding activity after exposure to heat. Heterozygous affected subjects (III-8 and I-2) have IEF patterns that combine the shifted bands and those of TBGC. Only the shifted bands of the variant TBGs in these sera lost ${ }^{125} \mathrm{I}-\mathrm{T} 4$ binding after heat treatment. ${ }^{125} \mathrm{I}$-T4-activity cathodal to $\mathrm{pH} 4.6$ represents T4binding to other serum proteins with lower affinity, mainly T4-binding prealbumin and albumin, visible in samples with reduced nTBG concentration and that become more prominent after the complete loss of nTBG. The distribution of ${ }^{125} \mathrm{I}-\mathrm{T} 4$ in heat-treated samples of hemizygous affected subjects is identical to that observed in unheated sera with complete TBG deficiency. sialic acid residues of TBG have a major contribution to the IEF pattern, which can be easily altered by partial desialylation (24, 25). Although the two new variant TBGs show variable shifts of $\mathrm{pl}$, there is full preservation of the multibanded IEF pattern. Furthermore, heat treatment equally denatures and inactivates T4 binding of all the IEF bands of TBG-Quebec and TBG-Montreal while the IEF bands of TBG-C and TBG-S, spanning over the same pl range, are preserved under the same treatment conditions. These findings suggest that the differences among various TBG types reside in the amino acid core of the molecule common to all IEF bands, and that secondary changes in the position of carbohydrate residues may be in part responsible for the observed IEF shifts.
Congenital TBG deficiency occurs in $\sim$ 1:7000 births (26) and about one-half show partial TBG deficiency. Our finding of altered TBG molecules in 2 of 16 families with partial TBG deficiency implies that inherited low serum TBG comprises a number of genetic defects, including structural gene mutations. Considering that in our study we selected only families with elevated dnTBG concentration, the number of mutant TBGs in the population must be very large. The benign nature of the defect may be the reason for the high degree of conservation and transmission of such variants to the progeny. Indeed, subjects with variant types of TBGs or altered serum TBG concentration are euthyroid, have normal survival and have no detectable handicaps $(1-7)$.

Table III. Summary of Pertinent Data on Variant TBGs in Humans*

\begin{tabular}{|c|c|c|c|c|c|c|}
\hline & \multirow{2}{*}{\multicolumn{3}{|c|}{ Serum levels (percentage of TBG-C) }} & \multicolumn{3}{|l|}{ nTBG properties } \\
\hline & & & & \multirow{2}{*}{$\begin{array}{l}\text { Heat lability } \\
\left({ }^{\circ} \mathrm{C} \text { at } t_{1 / 2}=7 \mathrm{~min}\right)^{\ddagger}\end{array}$} & \multirow{2}{*}{$\begin{array}{l}K_{\mathrm{a}} \text { for T4 } \\
\text { (\% of TBG-C) }\end{array}$} & \multirow{2}{*}{$\begin{array}{l}\text { IEF } \\
\text { (pI-shift) }\end{array}$} \\
\hline & nTBG & dnTBG & TT4 & & & \\
\hline TBG-C & 100 & 100 & 100 & 60 & 100 & - \\
\hline TBG-S & 88 & NL" & 84 & 59 & 100 & -0.06 \\
\hline TBG-A & 74 & NL & 58 & 58 & 54 & - \\
\hline TBG-Quebec & 16 & 260 & 41 & 50 & 70 & -0.06 \\
\hline TBG-Montreal & 14 & 390 & 38 & 48 & 73 & -0.02 \\
\hline TBG-Gary & 1.2 & 1,000 & 24 & 45 & $<5^{9}$ & +0.02 \\
\hline
\end{tabular}

* Data represent mean values of all hemizygous men with variant TBGs studied in our laboratory (references $10,11,13$, and 14, and this paper). Heat lability and IEF patterns were also compared in the same determination using a serum sample from each TBG variant (Figs. 4 and 8 ).

${ }^{\ddagger}$ Temperature at which $50 \%$ denaturation of the variant nTBG occurs in $\sim 7 \mathrm{~min}$. \$Sign preceding value refers to cathodal $(-)$ or anodal $(+)$ shift. "NL, normal or same as TBG-C when range but not precise mean value was available. 'Estimated, because accurate Scatchard plots could not be obtained due to marked reduction of T4-binding and TBG concentration. 


\section{Acknowledgments}

We are indebted to Drs. Y. Murata, D. Sarne, N. Scherberg, P. Ceccarelli, and $K$. Ain for their criticism and suggestions in the course of this work. We thank Drs. V. Chandramouli and J. S. Marshall for the provision of purified nTBG and RP-TBG and Mrs. Y. W. Richmond for the preparation of the manuscript.

This work was supported in part by United States Public Health Service grant AM 15070.

\section{References}

1. Robbins, J., S. -Y. Cheng, M. C. Gershengorn, D. Glinoer, H. J. Cahnmann, and H. Edelnoch. 1978. Thyroxine transport proteins of plasma. Molecular properties and biosynthesis. Recent Prog. Horm. Res. 34:477-519.

2. Refetoff, S. 1979. Thyroid function tests. In Endocrinology. Vol. 1. L. J. DeGroot, editor. Grune \& Stratton, Inc., New York. 387-428.

3. Gershengorn, M. C., D. Glinoer, and J. Robbins. 1980. Transport and metabolism of thyroid hormone. In The Thyroid Gland. M. DeVisscher, editor. Raven Press, New York. 81-121.

4. Beierwaltes, W. H., and J. Robbins. 1959. Familial increase in the thyroxine-binding sites in serum alpha globulin. J. Clin. Invest. 38:16831688.

5. Refetoff, S., N. I. Robin, and C. A. Alper. 1972. Study of four new kindreds with inherited thyroxine binding globulin (TBG) abnormalities. Possible mutations of a single gene locus. J. Clin. Invest. 51:848-867.

6. Refetoff, S., V. S. Fang, J. S. Marshall, and N. I. Robin. 1976. Metabolism of thyroxine-binding globulin in man. Abnormal rate of synthesis in inherited thyroxine-binding globulin deficiency and excess. J. Clin. Invest. 57:485-495.

7. Burr, W. A., D. B. Ramsden, and R. Hoffenberg. 1980. Hereditary abnormalities of thyroxine-binding globulin concentration. $Q$. J. Med. 49:295-313.

8. Dick, M., and F. Watson. 1981. A possible variant of thyroxinebinding globulin in Australian Aborigines. Clin. Chim. Acta 116:361357.

9. Daiger, S. P., D. P. Rummel, L. Wang, and L. L. Cavalli-Sforza. 1981. Detection of genetic variation with radioactive ligands. IV. Xlinked, polymorphic genetic variation of thyroxin-binding globulin (TBG). Am. J. Hum. Genet. 33:640-648.

10. Refetoff, S., and Y. Murata. 1985. X-chromosome-linked inheritance of the variant thyroxine-binding globulin in Australian Aborigines. J. Clin. Endocrinol. \& Metab. 60:356-360.

11. Takamatsu, J., M. Ando, M. Weinberg, and S. Refetoff. 1986. Isoelectric focusing variant thyroxine-binding globulin in American Blacks: increased heat lability and reduced serum concentration. J. Clin. Endocrinol. \& Metab. 63:80-87.
12. Grimaldi, S., L. Bartalena, C. Ramacciotti, and J. Robbins. 1983. Polymorphism of human thyroxine-binding globulin. J. Clin. Endocrinol. \& Metab. 57:1186-1192.

13. Murata, Y., S. Refetoff, S. H., Sarne, M. Dick, and F. Watson. 1985. Variant thyroxine-binding globulin in serum of Australian Aborigines: its physical, chemical and biological properties. J. Endocrinol. Invest. 8:225-232.

14. Murata, Y., J. Takamatsu, and S. Refetoff. 1986. Inherited abnormality of thyroxine-binding globulin with no demonstrable thyroxinebinding activity and high serum levels of denatured thyroxine binding globulin. N. Engl. J. Med. 314:694-699.

15. Refetoff, S., Y. Murata, G. Vassart, V. Chandramouli, and J. S. Marshall. 1984. Radioimmunoassays specific for the tertiary and primary structures of thyroxine-binding globulin (TBG): Measurement of denatured TBG in serum. J. Clin. Endocrinol. \& Metab. 59:269-277.

16. Refetoff, S., and H. A. Selenkow. 1968. Familial thyroxine-binding globulin deficiency in a patient with Turner's syndrome (XO): genetic study of a kindred. N. Engl. J. Med. 278:1081-1087.

17. Horwitz, D. L., and S. Refetoff. 1977. Graves' disease associated with familial deficiency of thyroxine-binding globulin. J. Clin. Endocrinol. \& Metab. 44:242-247.

18. Refetoff, S., S. R. Hagen, and H. A. Selenkow. 1972. Estimation of the T4 binding capacity of serum TBG and TBPA by a single T4 load-ion exchange resin method: comparison to two unrelated methods, survey of the population and abnormalities in various disease. J. Nucl. Med. 13:2-12.

19. Robin, N. I., S. R. Hagen, F. Collaço, S. Refetoff, and H. A. Selenkow. 1971. Serum tests for measurement of thyroid function. Hormones (Basel). 2:266-279.

20. Amino, N., S. R. Hagen, N. Yamada, and S. Refetoff. 1976. Measurement of circulating thyroid microsomal antibodies by the tanned red cell hemagglutination technique: its usefulness in the diagnosis of autoimmune thyroid diseases. Clin. Endocrinol. 5:115-125.

21. Scatchard, G. 1949. The attractions of proteins for small molecules and ions. Ann. NY Acad. Sci. 51:660-672.

22. Bliss, C. I. 1967. Statistics in Biology. Vol. I. McGraw-Hill Book Co., New York, 1-558.

23. Murata, Y., J. A. Magner, and S. Refetoff. 1986. The role of glycosylation on the molecular conformation and secretion of thyroxine binding globulin. Endocrinology 118:1614-1621.

24. Gartner, R., R. Henze, K. Horn, C. R. Pickardt, and P. C. Scriba. 1981. Thyroxine-binding globulin: Investigation of microheterogeneity. J. Clin. Endocrinol. \& Metab. 52:67-664.

25. Däiger, S. P., and R. S. Wildin. 1981. Human thyroxine-binding globulin (TBG): heterogeneity within individuals and among individuals demonstrated by isoelectric focusing. Biochem. Genet. 19:673-685.

26. Dussault, J. H. 1985. An update on screening for congenital hypothyroidism. Thyroid Today 8:1-5. 